Fermilab

PBAR NOTE \# 463

\author{
COOLING OF STORED BEAMS \\ F. E. Mills \\ $6 / 10 / 86$
}




\section{COOLING OF STORED BEAMS}

F. E. Mills

Fermi National Accelerator Laboratory, Batavia, Illinois*

\section{ABSTRACT}

Beam cooling methods developed for the accumulation of antiprotons are being employed to assist in the performance of experiments in Nuclear and Particle Physics with ion beams stored in storage rings. The physics of beam cooling, and the ranges of utility of stochastic and electron cooling are discussed in this paper.

\section{INTRODUCTION: MIXING AND HEATING}

Since we are dealing with particles circulating in storage rings, we need to describe the properties of the orbits of the particles. We will install devices in the ring, which the particles will pass at their revolution frequencies $f=V / C$, where $V$ is the particle velocity, and $C$ is the length of the orbit. If we consider particles of differing momenta $\Delta P$, then both the particle velocities and the particle orbit lengths will differ, the latter due to the dispersion in the magnetic system which confines the particles. There will be a spread of revolution frequencies given by

$$
\Delta f / f=\Delta V / V-\Delta C / C \equiv\left(1 / \gamma^{2}-1 / \gamma_{t}{ }^{2}\right) \Delta P / P \equiv \eta \Delta P / P
$$

If $f_{0}$ is the central revolution frequency of the beam particles, and we detect the beam current at frequencies in the neighborhood of $\mathrm{nf}_{0}$, where $n$ is an integer, then we will see a spread of frequencies

$$
\Delta f=n f_{0} n \Delta P / P
$$

where $\triangle P / P$ is the fractional momentum spread of the beam. This signal is called the nth longitudinal Schottky line of the beam. At sufficiently high frequency, $\Delta f \rightarrow f_{0}$, the bands overlap, and the signal becomes uniform in frequency.

* Operated by Universities Research Association Inc., under contract with the U.S. Department of Energy. 
In order that the particles will stay in the ring, we configure the magnetic system such that particles not on their closed orbit will oscillate about it with a "betatron oscillation". Both the particle's position $x$ and its angle $x$ ' (with respect to its closed orbit) will oscillate with a frequency $u f_{0}$. If we now detect the beam position, or more precicely its dipole moment at some location in the ring, we see, for each particle, a periodic $\delta$-function modulated at frequency $\nu f_{0}$. Then the transverse Schottky bands appear at frequencies $(n \pm v) f_{0}$. In most cases of interest, the magnetic structure will be configured such that $\nu$ is independent of $P$ ("chromaticity" $\xi=0$ ), and that the dependence of $v$ on betatron oscillation amplitude is small. Then the width of the nth transverse Schottky line is just the width of the nth longitudinal line. We now need to introduce new variables $(1, \sigma)$ to describe the betatron oscillations. Let

$$
\begin{aligned}
& 1=\left(\gamma x^{2}+2 \alpha x x^{\prime}+\beta x^{\prime 2}\right) / 2, \quad \tan \sigma=\alpha+\beta x^{\prime} / x \\
& x=(2 \mid \beta)^{1 / 2} \cos \sigma, \quad x^{\prime}=(2 \mid / \beta)^{1 / 2}[\sin \sigma-\alpha \cos \sigma]
\end{aligned}
$$

where $(\alpha, \beta, \gamma)$ are the lattice parameters determined by the confinement sustem, and are periodic functions of position $s$ along the orbit ${ }^{1}$. The equations of motion are

$$
I^{\prime}=0, \sigma^{\prime}=1 / \beta
$$

We infer that $v=(\phi d s / \beta) / 2 \pi$.

We now inquire about beam heating, that is, we subject the beam to a deflection $\theta(t)$ at some location, where $\theta$ is a random function of time and has zero mean. Then we find on each passage

$$
\delta 1=(2 \beta \mid)^{1 / 2} \sin \sigma \theta+\beta \theta^{2} / 2, \delta \sigma=(\beta / 21)^{1 / 2} \cos \sigma \theta
$$

We next sum up the contributions from successive passages $l$ at times $t_{l}$ $=t_{0}+l / f_{0}$, and $\sigma_{l}=2 \pi f_{0} \nu t_{l}+\sigma_{0}$ during a long time interval $T$ and find the long term rates of change $d I /\left.d t \equiv \Sigma \delta\right|_{\ell} / T$ and $\left.d\right|^{2} / d t \equiv \Sigma\left(\left.\delta\right|_{\ell}\right)^{2} / T$ for a particle averaged over initial phases and times. We are not interested in the changes in $\sigma$. We find ${ }^{2}$

$$
\begin{aligned}
& d l / d t=\beta f_{0} \int P(f) d f \\
& d l^{2} / d t=\beta l f_{0}^{2} \sum P\left[f_{0}(n \pm v)\right]
\end{aligned}
$$

Here $P(f)$ is the spectral power density of $\theta$ ( in the sense that $\theta^{2}$ represents power $)^{3}$. The sum is over all transverse Schottky lines. The 
first moment, wide band heating, is typical of gas and target scattering, dipole magnet ripple, etc. The second moment will be important in stochastic cooling, where the beam itself provides noise at the frequencies of the Schottky lines. At this point we could introduce the Fokker-Planck equation to determine the evolution of the distribution of betatron amplitudes, but we will wait until we have introduced the cooling terms. The second moment term is typical of results we will obtain, where the effect on the beam particles is obtained by adding up the contributions of all Schottky bands in the bandwidth of the system in question.

\section{STOCHASTIC COOLING}

Consider the arrangement shown in Fig. 1. A single particle is circulating in a storage ring. A beam pickup detector sends its signal to

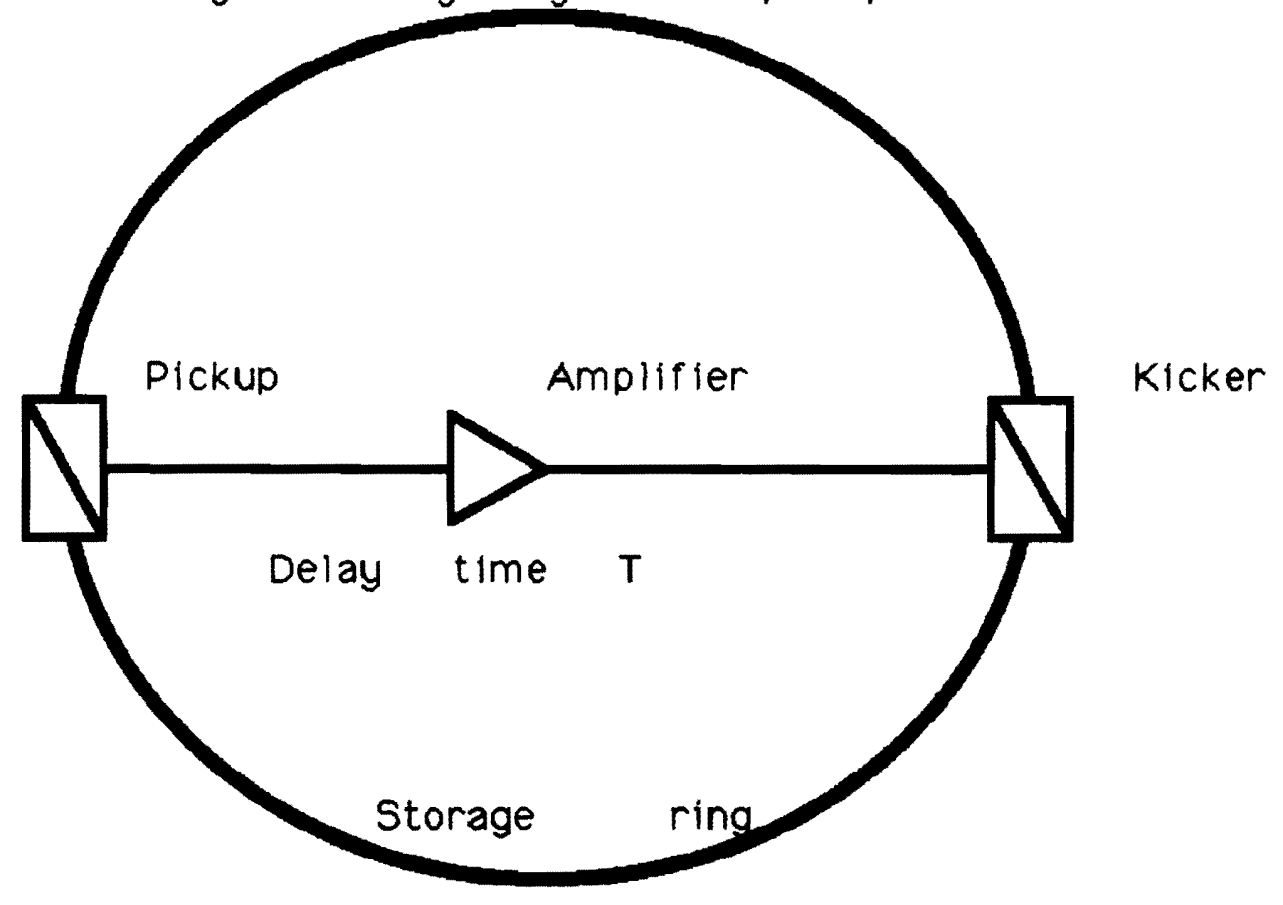

Figure 1. Stochastic cooling system schemati,

an amplifier, which drives a dipole kicker. The time delay of the signal $T$ is adjusted so that it equals the transit time of a particle with central momentum from pickup to kicker. The betatron phase shift 
between pickup and kicker is chosen to be near $\pi / 2$. As the particle passes through the system on successive revolutions, it will, on the average, receive a kick which reduces its betatron amplitude in proportion to its amplitude. The amplitude will fall exponentially. Amplifier noise will heat the particle at the same time, and the particle will approach a state of equilibrum between the heating and the cooling 4 .

The pulse sent to the kicker will have a nonzero time width because of the limited bandwidth of the system. If the particle has a momentum differing from the central momentum, it will arrive at the kicker at a different time than the signal, and will receive less kick, so its cooling rate will be reduced. The higher the bandwidth, the shorter the pulse, and the more important the effect. This is called "bad mixing" or "mixing between pickup and kicker". The effect of the high frequency Schottky bands can be reduced or even turned into heating by this effect. If we define $Y(f)$ to be the ratio, at frequency $f$, of the kick $\theta$ to the dipole moment at the kicker, then we can calculate the long term rate of change of the amplitude I of a particle due to the effect of the system (ignoring noise). It is,

$$
d l / d t=f_{0} \mid \sqrt{ }\left(\beta_{1} \beta_{2}\right) \sin \phi \sum\left[Y \cos \left(2 \pi n f_{0} \Delta t\right)\right] / 2 \pi
$$

The amplitude of $Y$, which contains the pickup sensitivity, the amplifier gain, and the deflection strength/volt of the kicker, has been taken to be constant in the bandwidth. $\phi$ is the betatron phase shift, the sum is over all longitudinal Schottky lines, and $\beta_{1}, \beta_{2}$ are the lattice functions at the pickup and kicker. $\Delta t$ is the particle transit time difference $T \eta \Delta P / P$, but can include unwanted amplifier phase shifts as well.

Now let us add more particles to the ring, say $N$ of them. Each particle will feel its own signal, and recelve cooling as in 2.1 . In addition it will feel the signal of all the others, and be heated according to 1.6. To estimate the heating, we need the power spectrum of the Schottky signal at the kicker. To obtain this, we find the power spectrum of the dipole moment of the beam $P_{D}$ at the pickup and multiply by $Y^{2}$. We find

$$
P_{D}(f)=\beta_{1} J N \sum \Psi\left[\left(f \pm v f_{0}\right) / n\right] / n(2 \pi)^{2}
$$

The sum is over all transverse Schottky lines, $J$ is the mean value of $I$ in 
the beam, and $\Psi$ is the distribution function of revolution frequencies $f_{0}$ in the beam. This is just the beam signal described above. As the frequency increases, the power density decreases until the bands overlap, and then remains constant. When all bands overlap, the situation is called "good mixing" in the sense that a fresh sample of beam is presented to the pickup on each revolution. We might further comment that in most situations of interest to this audience, namely intense beams, we can neglect amplifier noise. To get an estimate of the combined effect of the cooling and heating we employ the Fokker-Planck equation for the evolution of the distribution function $F(I)$ of amplitudes. The Fokker-Planck equation is essentially a continuity equation in which the particle flux $\Phi$ includes both the flux due to the cooling and the diffusive flux due to the heating ${ }^{5}$.

$$
\partial F / \partial t+\partial \Phi / \partial \mid=0 ; \Phi=(d \mid / d t) F-\left(\left.d\right|^{2} / d t\right)(\partial F / \partial \mid) / 2
$$

We multiply this equation by 1 and integrate over 1 to find the rate of change of $J$, the mean value of 1 . Historically the result has been written in the following useful way ${ }^{6}$;

$$
J / J=-(W / N)\left[g-g^{2} M / 2\right]
$$

Here $W$ is the bandwidth of the cooling system, $g$ can be interpreted as the fractional correction applied by the system on each revolution when it measures an apparent of fset of the beam ( $-\mathrm{gW} / \mathrm{N}$ is just the right hand side of eq. 2.2), and $M$ is the "mixing factor". The mixing factor is equal to one for good mixing, 1.e. all bands overlap. If no bands overlap, $M=\left[\Sigma f_{0} / \Delta f_{n}\right] / n_{l} \quad 2.5$ $\Delta f_{n}$ is the width of the $n$th Schottky band and $n_{b}$ is the number of Schottky lines in the bandwidth $W$ included in the sum. Clearly $M$ describes the effect of frequency spreads on the ratio of the heating term to the cooling term.

From equation 2.4 we see that optimum cooling takes place when $g=1 / M$, in which case the shortest cooling time is

$$
T_{\text {opt }}=2 M N / W
$$

Another effect appears when systems operate near optimum gain. The kicker signal induces coherent motion in the beam. This signal tends to reduce the spontaneous beam signal at the pickup, and to slow down the cooling. As a practical matter, this does not pose a much greater 
limitation than the heating term, and is intimately related to it, since both effects depend critically on the mixing. On the other hand, if the gain is set high, and the system has improper phase adjustment, instability can take place in the improperly adjusted bands.

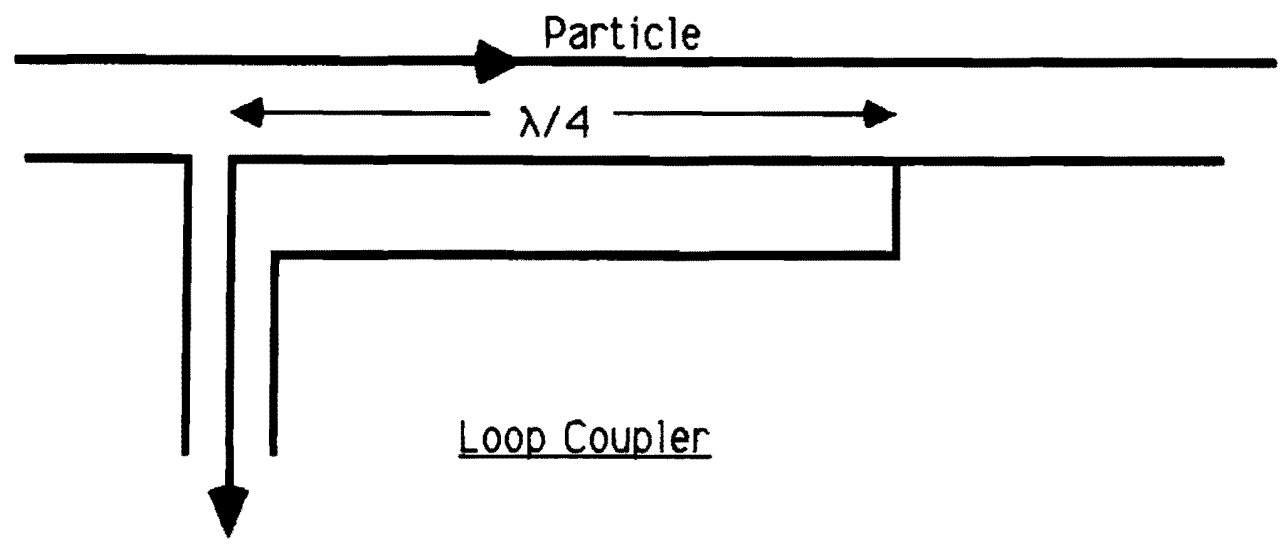

Signal out

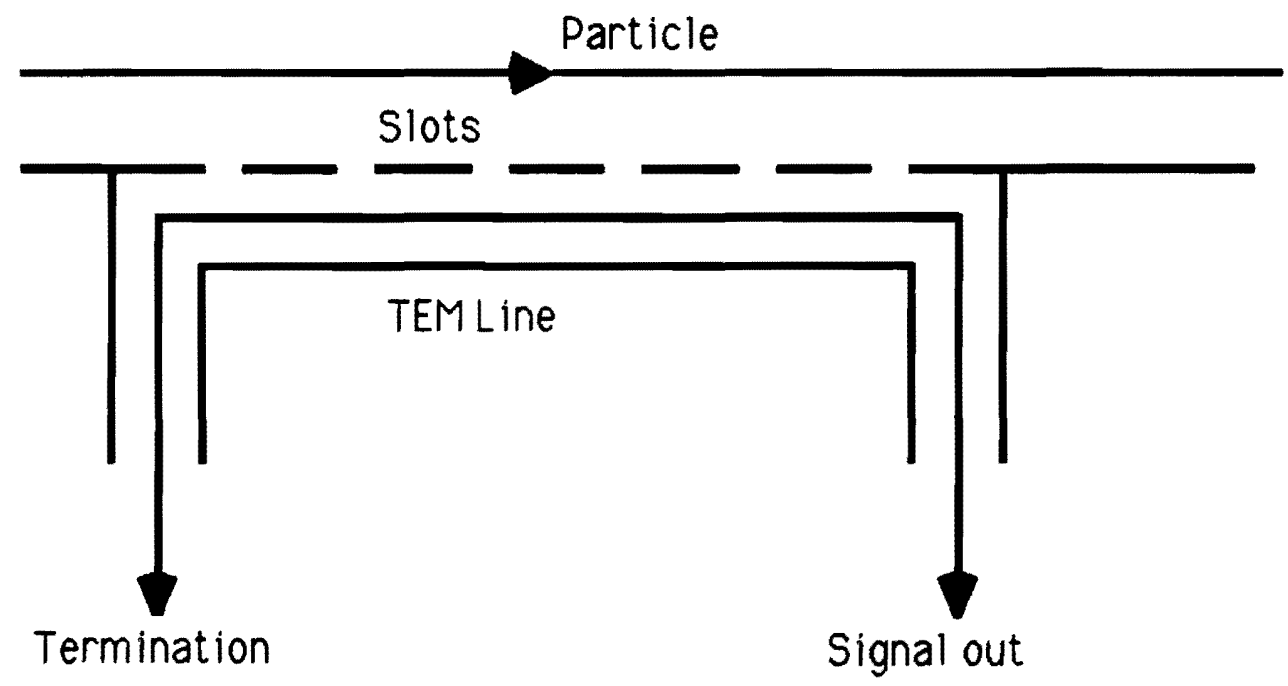

Slot coupler

Figure 2. Pickup and Kicker Geometries

Concerning hardware, loop couplers of length $\lambda / 4$ in midband seem to offer the maximum sensitivity for relativistic particles, although TEM lines coupled through slots to the beam find application at lower velocity, or in situations in which there is large signal. These pickups 
are shown pictorially in Fig. 2. Kickers tend to be the same structure, except for power handling capability, because of a reciprocity relationship. Special slowwave structures ( helices, lumped lines ) have been used in experiments. The signal from many loops can be combined to improve the signal to noise ratio. In the TeV-1 systems, the signals of 128 loops are combined. Travelling wave tubes with octave bandwidth have been employed for frequencies up to $2-4 \mathrm{gHz}$, and solid state amplifiers may soon be available. Output power requirements can be reduced by splitting the high level signal and driving many kickers. Again, TeV-1 systems drive 128 loop couplers ${ }^{8}$.

Stochastic cooling can be employed to cool momentum spread as well. The principal difference is that as the momentum spread is reduced, the Schottky heating power density is increased, and the cooling slows down. Special filters have been employed to shield the beam, in frequency, from its dense core and to allow cooling of hotter particles $^{9}$.

We can use Eq. 2.6 to estimate the utility of stochastic cooling as the primary cooling means for a ring such as the Indiana Cooler. For high precision experiments it would be desireable to have $\triangle P / P=10^{-4}$, say $\eta=.5, f_{0}=10^{6} \mathrm{~Hz}, N=10^{10}$, and $W=10^{9} \mathrm{~Hz}$. Then $M=20$, and $T_{\text {opt }}=400$ $\mathrm{sec}$. The momentum cooling system to obtain $\Delta p / p=10^{-4}$ would be very difficult, with many pickups and kickers, which would have to be delay-adjusted to cool different particle velocities. Nevertheless, stochastic cooling will probably find use in such machines. As we shall see below, cooling of large amplitudes is very slow for electron cooling systems. Scattering from internal targets will, in some circumstances, create a beam halo which will cause backgrounds which are undesireable. Betatron cooling systems are being contemplated for LEAR in which the pickup electrodes have spatially varying sensitivity so that the system responds to the halo and not to the dense core ${ }^{10}$. Similar systems are under study at Fermilab for the Tevatron collider, where normal cooling systems would have cooling times of days for the intense bunched beams. In colliders, the beam-beam tune shift provides some frequency separation between the core and the halo. This aids the situation, since the halo signal will not heat the core. 


\section{ELECTRON COOLING}

Consider a system of spatially coincident ion and electron beams which have the same mean velocity vectors. Of course this cannot be true for the whole circumference of the storage ring, so we will be speaking of what happens in some fraction $\eta$ of the ring, called the cooling region. The means for bringing the two beams together will be discussed below. Now consider the two beams from the point of view of an observer moving at the beams mean velocity. He will see an ion gas and an electron gas. If the ion gas is hotter than the electron gas, the ions will lose energy to the electrons by Coulomb collisions and tend to come into temperature equilibrium with the electron gas 11,12 Kilovoit ions come to rest in condensed matter in times of the order of $10^{-13} \mathrm{sec}$, so that in an electron beam whose electron density is of order $10^{-11}$ that of condensed matter, we might expect that equilibrium would come in some milliseconds, or seconds considering the fraction $\eta$ of the time the ion is actually in the electron beam.

since the process is coulomb scattering, the rates will be determined by the accessible range of impact parameters and the relative velocities of the two species. Electron guns for this purpose have operated in a longitudinal magnetic field of about 1 kilogauss, and either provide a beam of constant diameter, or increase the field as the electrons move from the cathode, in which case the beam diameter decreases and the temperature increases. For our purposes, let us consider a beam of $5 \mathrm{Amp}, 5 \mathrm{~cm}$ diameter, $T_{e}=1 \mathrm{eV}$, and kinetic energy of $110 \mathrm{keV}$. Some important parameters are then;

$\begin{array}{lll}\text { density } & n & 1 \cdot 10^{8} \mathrm{~cm} \\ \text { plasma frequency } & \omega_{\mathrm{p}} & 6 \cdot 10^{8} \mathrm{~Hz} \\ \text { cyclotron frequency } & \omega_{\mathrm{c}} & 2 \cdot 10^{10} \mathrm{~Hz} \\ \text { electron } \perp \text { velocity } & \beta_{\mathrm{e} \perp} \approx \sqrt{ }\left(2 \mathrm{~T}_{\mathrm{e}} / \mathrm{mc}^{2}\right) & 2 \cdot 10^{-3} \\ \text { Debye length } & \lambda=\sqrt{\left(\mathrm{T}_{\mathrm{e}} / 4 \pi \mathrm{ne}^{2}\right)} & 7 \cdot 10^{-2} \mathrm{~cm} \\ \text { electron gyroradius } & \rho_{\mathrm{e}} & 3 \cdot 10^{-3} \mathrm{~cm} \\ \text { minimum approach } & \mathrm{b}_{\min } & 1.4 \cdot 10^{7} \mathrm{~cm} .\end{array}$

When the electrons are accelerated, their tranverse momenta, and hence their transverse temperature, tend to remain constant or to be 
regulated by the magnetic field as noted above. In the longitudinal direction, however, the electron temperature is compressed by the acceleration process, $(\Delta E=m v \Delta v)$, and the electron parallel velocity becomes $\beta_{\mathrm{el}}=T_{\text {cath }} / C P_{e}=3 \cdot 10^{-6}$. Then the electron velocity distribution in the moving system is far from Maxwellian, rather resembling a disc. For impact parameters above $\lambda$ the electric field of the ion is screened by the intervening electrons. Collisions taking place for impact parameters between $b_{\min }$ and $\rho_{e}$ are normal free-free coulomb collisions, exactly the same as those considered in stopping power in normal matter. Collisions for impact parameters between $\rho_{e}$ and $\lambda$ are adiabatic with respect to the electron gyromotion, and no momentum can be transferred perpendicular to the field lines. This means that the only electron velocity important in the collision is the parallel one, and it is very small. If the ion velocity is small also, the cooling can be very fast. For the nonadiabatic collisions, the electron $\perp$ velocity normally determines the cooling rates. Then there are two Coulomb logarithms $\wedge$, the nonadiabatic one of about 10 , and the adiabatic one of about 3 .

Space charge also contributes to the electron velocity spreads. The space charge potential of the e-beam $V=30.1 / \beta \approx 200 \mathrm{eV}$ causes a spatially correlated $\|$ electron velocity spread. The same radial electric field, combined with the magnetic field, causes the e-beam to rotate, typically .05-.1 rad $/ \mathrm{m}$.

The ion velocities are determined by the emittance and momentum spread of the ion beam. Since the $\perp$ momentum is invariant, the ion $\perp$ velocity is given by the ion angle, $\beta_{i \perp}=(\beta \gamma) x$. To have the same $\beta_{\perp}$ as the e-beam the ion emittance would be $[(\beta \gamma)=7$, lattice $\beta=20 \mathrm{~m}] \epsilon / \pi=$ $100 \mathrm{~mm} \mathrm{mr}$, which is not restrictive. In the II direction, $\beta_{1}=\beta \Delta P / P$, so $1 \sigma^{3}$ is typical. Two other transfomations between laboratory and moving system are required by Lorentz invariance, affecting clock rates and densities: $n_{\text {moving }}=n_{\text {lab }} / \gamma$, and $\Delta t_{\text {lab }}=\Delta t_{\text {moving }} / \gamma$. Both of these are in the sense to reduce cooling rates as the beams become relativistic.

In order to calculate cooling rates, we must add up the momentum tranfers of collisions of all impact parameters and all electron velocities as an ion moves through the electron gas. There is an analogy, for the nonadiabatic collisions, between the ion friction force due to the 
electron velocity distribution, and the electric field due to a similar charge distribution. Because of the multiparameter nature of the process, it is customary to treat the $\|$ and $\perp$ cooling separately. For the II cooling, because of the disc nature of the electron distribution, the friction force is slowly varying for $\beta_{\mathrm{e}}<\beta_{\mathrm{il}}<\beta_{\mathrm{e} \perp}$ and then falls as $1 / \beta_{i}{ }^{2}$ for larger velocities (as does the transverse friction force). The ॥ friction force is a good measure of the ability to cool a beam, and to restore energy losses due to targetry. For the above parameters, with the fractional cooling length $\eta=.04$ we can estimate the rate of reduction of momentum spread $\delta \equiv \triangle P / P$ to be

$$
\delta^{\prime}=-8 \pi n c m_{e} c^{2} r_{e}^{2} \wedge \eta /\left[\left(m_{j} / m_{e}\right) \beta \gamma^{2} T_{e}\right] \approx 10^{-3} \mathrm{~Hz}
$$

For transverse cooling, when $\beta_{\perp i}<\beta_{\perp e}$ the friction force is proportional to velocity so there is a characteristic cooling time

$$
T=\left(T_{e \perp} / m_{e} c^{2}\right)^{3 / 2} \gamma^{3} /\left[2 \pi n r_{e} r_{i} c \wedge \eta\right] \approx 12 \mathrm{sec}
$$

For smaller ion emittances the adiabatic or magnetic cooling can reduce the cooling time appreciably. There is a substantial premium to be gained by starting with the smallest possible emittance and momentum spread. The magnetic friction forces are;

$$
\begin{aligned}
& F_{\perp}=-\left[8 \pi n e^{4} \wedge \eta / m_{e}\right]\left[v_{\perp}^{2} 2-2 v_{1}^{2}\right] v_{\perp} / v^{5} \\
& F_{1}=-\left[6 \pi n e^{4} \wedge \eta / m_{e}\right] v_{\perp}{ }^{2} v_{1} / v^{5}
\end{aligned}
$$

Here $\left(v, v_{\perp}, v_{n}\right)$ refer to the ion velocities in the moving system, as do the friction forces $F$, and the density $n$. $\wedge$ is here the adiabatic coulomb logarithm, equal to about 3 . Note that the parallel force is zero when the ion moves parallel to the field, and that the ion velocity vector is first rotated to a more $\perp$ direction before its magnitude is reduced.

There is no experience in the electron cooling of very intense beams, say for more than $10^{9}$ particles. One can observe, however, that the cooling will reduce the frequency spreads in the beams and render them susceptible to instabilities. In the past means have usually been available to control these instabilities when they occur.

It is beyond the scope of this report to describe completely the technological basis of cooling systems. Suffice it to say that guns for the currents and temperatures required have been constructed, and that newer designs incorporate significant improvements. The use of toroidal fields to bring the ion and electron beams together works well, 
and efficient electron collection systems have been built with $97 \%$ energy recovery efficiency and $10^{-4}$ electron recovery inefficiency. Adequate methods have been developped to sweep unwanted trapped lons from the electron beams, and suitable vacuum practices now exist to be consistent with long beam storage times $13,14,15$

\section{CONCLUSION}

Modern cooling techniques are directly applicable to Nuclear and Particle Physics Experiments utilizing cooled stored beams and internal targets. Altogether about 8-10 cooling rings are being planned in Europe, North America, and Asia for these purposes and for Atomic Physics experiments. This experimental medium promises high resolution, low backgrounds, and adequate luminosity for many types of experiments. To achieve all these goals, probably both electron cooling and stochastic cooling will be required.

\section{REFERENCES}

1. Courant, E., Snyder, H., Annals of Physics 3 , 1 (1958)

2. Cole, F.T., Mills, F.E., Ann. Rev. Nucl. Part. Sci. 31, 295(1981)

3. Wiener, N., Time Series, MIT Press (Cambridge 1949), p. 37

4. van der Meer, S., Stochastic Damping of Betatron Oscillations in the ISR. CERN/ISR-PO/72-31

5. Chandrasekhar, S., Stochastic Problems in Physics and Astrometry. In " Selected Papers on Noise and Stochastic Processes ", Dover (New York 1954), p. 3-93

6. Sacherer, F., Stochastic Cooling Theory, CERN-ISR-TH/78-11

7. Lambertson, G.R., et. al., Stochastic Cooling of $200 \mathrm{MeV}$ Protons, Proc. 1 ith Int. Conf. High Energy Accelerators, Birkhauser (Basel 1980), p.794

8. Design Report Tevatron 1 Project, Fermilab (Batavia 1984), p. 5-12

9. Carron, G., Thorndahl, L., Stochastic Cooling Of Momentum Spread by Filter Techntques, CERN/ISR-RF/78-12

10. Mohl, D., Private Communication, 1986 
11. Budker, G.I. et. al, Proc. Int. Symp. Electron and Positron Storage Rings, Atomnaya Energiya 22, 346 (1967)

12. Derbenev, Y.S., Skrinsky, A.N., Preprint INP-225, (Novosibirsk 1968)

13. Budker, G.I., et. al., Part. Accel. Z, 197(1976)

14. Krienen,F., Proc. 11 th Int. Conf. High-Energy Accelerators, Birkhauser (Basel 1980), p. 781

15. Ellison, T., et. al., Electron Cooling and Accumulation of $200 \mathrm{MeV}$ Protons at Fermilab, IEEE Trans Nuc. Sci., NS-30, 2636 (1983) 